\title{
Colliding galaxies and the rapid assembly of clouds into proto-super star clusters
}

\author{
Guillermo Tenorio-Tagle, ${ }^{1}$ Enrique Pérez, ${ }^{2}$ Casiana Muñoz-Tuñón, ${ }^{3}$ \\ Sergiy Silich ${ }^{1}$ and Jan Palouš ${ }^{4}$ \\ ${ }^{1}$ Instituto Nacional de Astrofísica Optica y Electrónica, AP 51, 72000 Puebla, Mexico \\ email: gtt@inaoep.mx \\ ${ }^{2}$ Instituto de Astrofísica de Andalucía (CSIC), Camino bajo de Huetor 50, E-18080 Granada, \\ Spain \\ ${ }^{3}$ Instituto de Astrofísica de Canarias, E-38200 La Laguna, Tenerife, Spain \\ ${ }^{4}$ Astronomical Institute, Academy of Sciences of the Czech Republic, Boční II 1401, \\ 14131 Prague, Czech Republic
}

\begin{abstract}
We present a short summary of several 2D hydrodynamic calculations that suggest that upon the collision of galaxies two physical mechanisms lead to the formation of protosuper star clusters. These are condensation, induced by radiative cooling, and implosion caused by the shocked intercloud medium. Even in the absence of gravity, these lead to storage and compression of the dense cloud component into massive and compact gravitationally unstable condensations. The resulting entities exhibit enhanced surface densities that are several hundred times higher than their initial values. These are here postulated as the cradles of very efficient and rapid star-formation episodes, able to withstand the negative feedback effects associated with star formation, while leading to the formation of massive and compact super star clusters.
\end{abstract}

Keywords. galaxies: star clusters, galaxies: interactions

\section{Introduction}

Hubble Space Telescope observations (Ho 1997; Melo et al. 2005; Gilbert \& Graham 2007; Walcher et al. 2005) led to the discovery of massive, young and compact collections of stars, today called super star clusters, the most spectacular modality of star formation. They are found in a large variety of galaxies, from blue compact dwarfs to colliding spirals. Their stellar masses range from a few $\times 10^{5}$ to several $\times 10^{6} \mathrm{M}_{\odot}$, all within a small volume of just a few pc across. Their discovery led immediately to a large number of questions. Perhaps the most important among these is (Tenorio-Tagle et al. 2003) how to collect all of the interstellar matter that will be turned into stars into such a small volume? Giant molecular gas complexes, with masses from several $\times 10^{5}$ to a few $\times 10^{6} \mathrm{M}_{\odot}$ soon became the favorite suspect sites where super star clusters could form. However, it is well known that molecular clouds far exceed the dimensions of super star clusters and so the question remains unanswered. How can so many stars form unaffected by the negative feedback produced by their neighboring stars? The question points particularly to massive stars. All stars with masses greater than $20 \mathrm{M}_{\odot}$ evolve rapidly while dumping an enormous amount of ionizing radiation into interstellar space, capable of heating the collapsing cloud, and thus of inhibiting star formation. Furthermore, all stars more massive than $8 \mathrm{M}_{\odot}$ exhibit strong stellar winds and end up exploding as supernovae. The worry is that such a large and continuous mechanical energy deposition may rapidly disrupt the 
collapsing cloud and inhibit further star formation. Thus, the central question today is how to avoid the negative feedback produced by massive stars or, alternatively, how do super star clusters form?

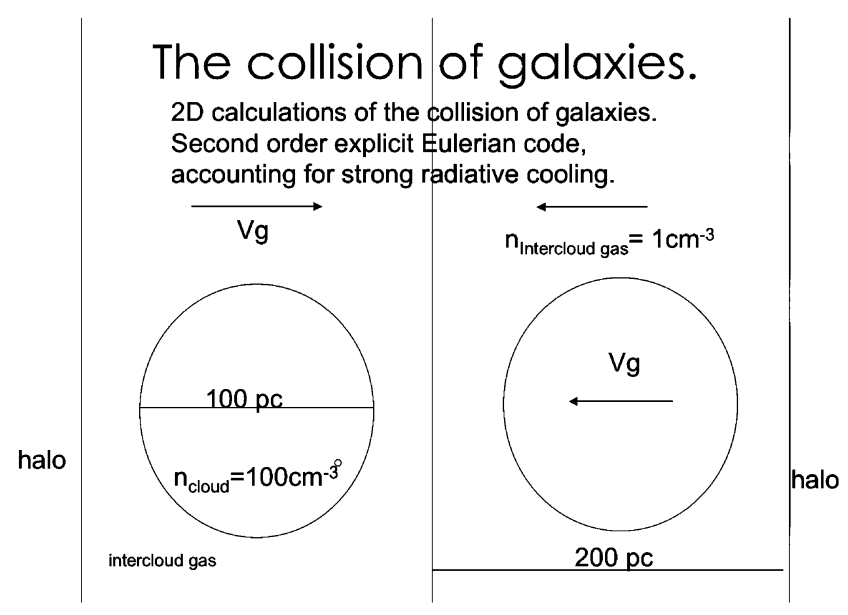

Figure 1. Input model. Two galactic disks approaching each other at high speed $\left(V_{\mathrm{g}}\right)$, with their molecular clouds set to undergo a head-on collision. The dimensions and assumed geometry are indicated in the figure.

\section{The model}

Based on the empirical evidence from the Antennae system (which now presents more than 1000 young super star clusters) and other colliding and merging galaxies (Whitmore et al. 1993; Gilbert \& Graham 2007), we have started a series of numerical simulations that consider direct collisions of molecular clouds and their surrounding intercloud medium. The calculations assume a collection of molecular clouds immersed in the disks of their host galaxies. The galaxies approach each other and interact at high speed, which leads to the appearance of two strong shocks that process both the intercloud medium and the molecular clouds. Our first results showed that dense clouds can cool rapidly through radiation, long before they are fully processed by their respective shock. On the other hand, if the intercloud gas is initially of low density, say, less than 1 atom $\mathrm{cm}^{-3}$, then its cooling time will be much longer than the collision time $\left(\sim 2 \times 10^{5} \mathrm{yr}\right)$. In this case, the shocked intercloud medium would remain hot and highly pressurized, and thus it would be able to implode both the as yet unprocessed cloud and the condensations or fragments that result from the direct cloud-cloud collision. The final result is a pile up of all initial cloud material into massive, compact condensations.

This all happens in a time that is much shorter than the gravitational collapse time of the original clouds. The timescale is also much shorter than the time required for the most massive stars to become supernovae and sufficiently short to allow production of only a small fraction of the total ionizing radiation expected from massive clusters. Star formation should then be able to proceed unaffected by negative feedback. This would lead to a very efficient star-formation episode.

However, this would only happen if a large-scale disturbance of the galaxies' interstellar medium can rapidly aggregate all of the required matter. Or, as phrased by Larson (1987), "If the gas fueling a starburst is turned into stars on a short 
timescale, then the gas must also be assembled into the starburst region in a similar timescale."

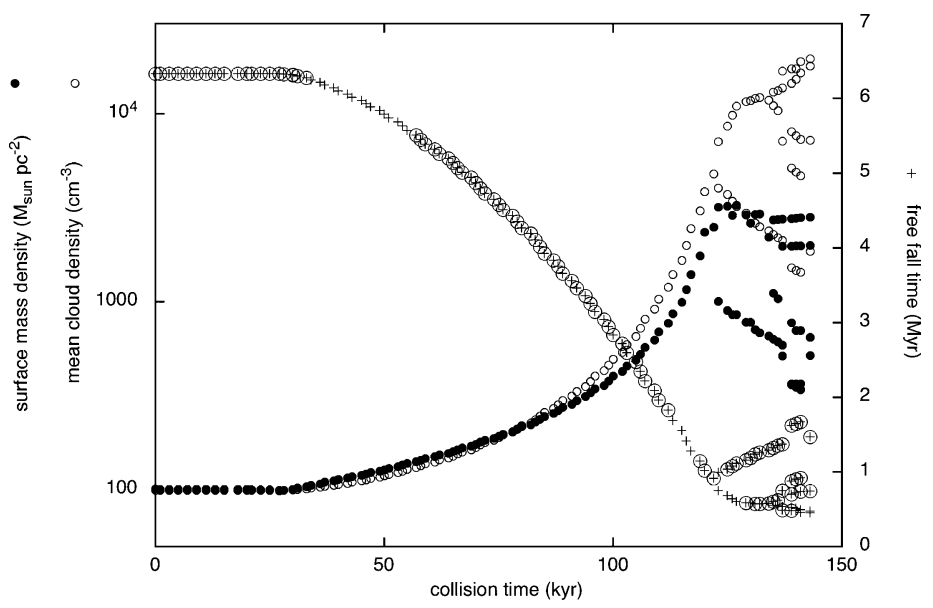

Figure 2. Rapid assembly of the cloud component into super star cluster building blocks. The left axis indicates surface mass density $\left(\mathrm{M}_{\odot} \mathrm{pc}^{-2}\right.$; filled circles) and the cloud's mean particle density $\left(\mathrm{cm}^{-3}\right.$; open circles $)$. The right axis marks the corresponding free-fall time $\left(\tau_{\mathrm{ff}}\right.$ in $\mathrm{Myr}$; encircled plus signs indicate Jeans-unstable clouds), all shown as a function of time. A few very small clouds have not been plotted for reasons of clarity.

\section{First results}

Figure 1 shows the initial condition for one of our 2D calculations. In this case, the clouds with a diameter of $100 \mathrm{pc}$ and a density of $100 \mathrm{~cm}^{-3}$ (and thus a mass of $1.2 \times$ $10^{6} \mathrm{M}_{\odot}$ each), are located in the plane of their respective galaxy. They are surrounded by an intercloud medium with a density of $1 \mathrm{~cm}^{-3}$ that extends to $100 \mathrm{pc}$ on either side of the galactic planes and all the way to the edge of the computational grid in the direction parallel to the plane of the interaction. The initial setup is such that the clouds will suffer a head-on collision, with an intensity defined only by the velocity of approach of the two galaxies, here $1000 \mathrm{~km} \mathrm{~s}^{-1}$. The calculations were performed using a 2D secondorder explicit Eulerian code, which accounts for strong radiative cooling (Tenorio-Tagle \& Muñoz-Tuñón 1997, 1998). The code does not account for gravitational terms and so the resulting flows are only due to radiation hydrodynamics.

Figure 2 plots the run of density $\left(\mathrm{cm}^{-3}\right)$, surface density $\left(\mathrm{M}_{\odot} \mathrm{pc}^{-2}\right)$ and free-fall time $\left(\tau_{\mathrm{ff}}=(G \rho)^{-1 / 2}=2 n_{3}^{-1 / 2} \mathrm{Myr}\right.$; where $n_{3}$ is the number density in units of $\left.10^{3} \mathrm{~cm}^{-3}\right)$ as a function of the evolution time (in kyr) for all clouds $\left(n \geqslant 100 \mathrm{~cm}^{-3}\right)$ recognized within the computational grid. The figure also indicates clouds that become Jeans unstable to gravitational collapse whenever their $\tau_{\mathrm{ff}}$ is shorter than their sound-crossing time $\left(\tau_{\mathrm{s}}=11 r T^{-1 / 2} \mathrm{Myr}\right.$, where $r$ is the fragment size in pc and $T$ their mean temperature, in degrees $\mathrm{K})$.

The figure shows how the resulting condensations end up with densities enhanced by a factor of between 10 and several hundreds from their original value. The same happens to their surface density, which is enhanced by factors of a few to several tens from the initial value and, correspondingly, the collapse time is drastically reduced from the initial value $(6.2 \mathrm{Myr})$ to a few $\times 10^{5} \mathrm{yr}$. The latter implies that during the collision time $(2 \times$ $10^{5} \mathrm{yr}$ ), the hydrodynamical events act much faster than gravity, leaving all of the dense 
gas in place within dense and compact condensations ready to collapse, fragment and form stars within a few $\times 10^{5} \mathrm{yr}$.

\section{Conclusions}

Given the structure of the interstellar medium and all of its different phases, one of the main questions regarding super star clusters is how to store the large amount of interstellar matter that is turned into stars into such compact volumes.

We have shown that the strong compression caused by the collision of galaxies and, in particular, direct cloud collisions, condensation and fragmentation induced by strong radiative cooling and implosion driven by the hot, shocked intercloud gas, lead to storage and compression of the dense cloud component into massive and compact gravitationally unstable condensations.

The time required to do so is about $10^{5} \mathrm{yr}$, much shorter than the initial collapse time of the individual clouds $\left(\tau_{\mathrm{ff}} \sim 6.25 \times 10^{6} \mathrm{yr}\right)$. This implies that a large-scale disturbance of a galaxy's interstellar medium may collect and compress the matter required to build super star clusters much faster than gravity alone. In our case, the large-scale disturbance is provided by a galaxy collision. The time required to assemble the cloud component into super star cluster building blocks is also similar to the final free-fall time of the resulting condensations (a few $\times 10^{5} \mathrm{yr}$ ). Having all matter in place on such short timescales has profound implications for feedback. This is because the assembly time is much shorter than the time when the first supernova (originating from the most massive star expected to form in the cluster) occurs. On this short timescale, only a small fraction of the total ultraviolet radiation expected from a massive cluster is produced. Star formation under such conditions can then proceed freely, without being affected by negative star-formation feedback, leading instead to a very efficient star-formation episode and possibly to the formation of a super star cluster.

Several more calculations including a structured interstellar medium in the disks and halos of the colliding galaxies, as well as grazing clouds, will be the subject of a forthcoming article.

\section{Acknowledgements}

This study was supported by CONACYT México under research grants 60333 and 82912, by the Spanish Ministry of Science and Innovation under grant AYA2007-64712, the ESTALLIDOS collaboration (grant AYA2007-67965-C03-01) and Consolider-Ingenio 2010 program grant CSD2006-00070: First Science with the GTC. The authors acknowledge support from the Institutional Research Plan AV0Z10030501 of the Academy of Sciences of the Czech Republic and from the project LC06014 Center of Theoretical Astrophysics of the Ministry of Education, Youth and Sports of the Czech Republic as well as the CONACYT Mexico and Czech Academy of Sciences research grant 2009-2010.

\section{References}

Gilbert, A. \& Graham, J. 2007, ApJ, 668, 168

Ho, L. C. 1997, Rev. Mex. A\&SA Conf. Ser., 6, 5

Larson, R. B. 1987 in: T. X. Thuan, T. Montmerle \& J. T. T. Van: Starbursts and galaxy evolution, p. 467, Gif-sur-Yvette: Editions Frontières

Melo, V. P., Muñoz-Tuñón, C., Maíz-Apellániz, J., \& Tenorio-Tagle, G. 2005, ApJ, 619, 270 
Tenorio-Tagle, G. \& Muñoz-Tuñón, C. 1997, ApJ, 478, 134

Tenorio-Tagle, G. \& Muñoz-Tuñón, C. 1998, MNRAS, 293, 299

Tenorio-Tagle, G., Palouš, J., Silich, S., Medina-Tanco, G. A., \& Muñoz-Tuñón, C. 2003, Aש্̇A, 411,397

Walcher, C. J., van der Marel, R. P., McLaughlin, D., Rix, H.-W., Böker, T., Häring, N., Ho, L. C., Sarzi, M., \& Shields, J. C. 2005, ApJ, 618, 237

Whitmore, B. C., Schweizer, F., Leitherer, C., Borne, K., \& Robert, C. 1993, AJ, 106, 1354 